\title{
Eine Verallgemeinerung eines Satzes von Kurata
}

\author{
Von Dirk Windelberg in Hannover
}

In [4], Theorem 3. 6 hat Y. Kurata 1965 in einem nicht-kommutativen und nichtassoziativen Ring $R$, der die Maximalbedingung für zweiseitige Ideale erfüllt, zu der Aussage: „Jedes zweiseitige Ideal in $R$ kann als Durchschnitt endlich vieler rechtsprimärer zweiseitiger Ideale aus $R$ dargestellt werden" vier äquivalente Bedingungen angegeben.

In [11] konnte ich zeigen, daß sich diese vier Bedingungen auch in der allgemeinen

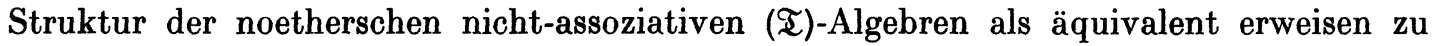
der in dieser Struktur formulierten Aussage: „Jedes Element einer noetherschen nichtassoziativen (I)-Algebra (L) kann als Durchschnitt endlich vieler linksprimärer Elemente aus $(L)$ dargestellt werden."

Inzwischen wurden für die Darstellungssätze von V. A. Andrunakievic und Ju. M. R. Jabuhin in [1] noch weiterführende Strukturen gefunden und dort ,Systeme mit Residuen" genannt. - Ich möchte hier für (T)-Systeme, die ebenfalls eine Verallge-

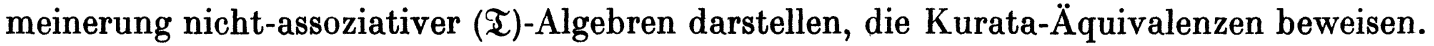

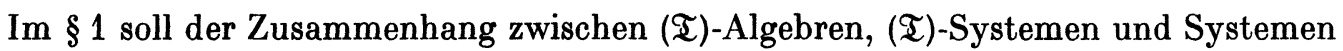
mit Residuen dargestellt werden ([1], [6] und [11]). Nach der Definition dieser Begriffe werden dann im $\S 2$ tertiäre und primäre Elemente in $(\mathfrak{T})$-Systeme eingeführt und einige die Bedeutung dieser Elemente erläuternde Sätze bewiesen.

Schließlich enthält der $\S 3$ den für die Kurata-Äquivalenzen so wichtigen Begriff der verallgemeinerten Bedingung von Artin und Rees ([7] und [8]), der hier so formuliert wurde, daß er die Gültigkeit eines zu dem oben erwähnten Satz Kuratas in [4] verallgemeinerten Satzes sichert; dieser Satz ist dann in $\S 4$ formuliert.

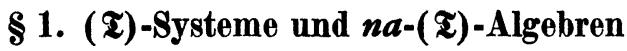

In [11], Definition 1. 4 hatte ich $n a-(\mathfrak{T})$-Algebren in folgender Weise definiert:

Definition 1. 1. Eine Menge zweier Verbände (I) und $(L)$ heißt na-(I)-Algebra $(L)$, falls die folgenden Bedingungen erfüllt sind:

(A) (I) ist ein quasi-ganzes und vollständiges Verbandsgruppoid ${ }^{1}$ ),

(B) $(L)$ ist ein vollständiger Verband,

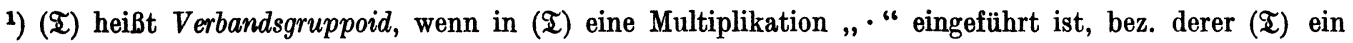
Gruppoid bildet und für die gilt $\mathfrak{A} \cdot(\mathfrak{B} \cup \mathfrak{C})=\mathfrak{A} \cdot \mathfrak{B} \cup \mathfrak{A} \cdot \mathfrak{C}$ sowie $(\mathfrak{A} \cup \mathfrak{B}) \cdot \mathfrak{C}=\mathfrak{A} \cdot \mathfrak{C} \cup \mathfrak{B} \cdot \mathfrak{C}$.

(I) heiBt quasi-ganz, falls gilt $\mathfrak{A} \cap \mathfrak{B} \geqq \mathfrak{A} \cdot \mathfrak{B} \forall \mathfrak{A}, \mathfrak{B} \in(\mathfrak{T})$,

(I) heißt vollständig, wenn $(\mathfrak{I})$ als Verband vollständig ist. 
(C) Zwischen (I) und $(L)$ ist eine Operation $\cdot:(\mathfrak{T}) \times(L) \rightarrow(L)$ erklärt mit

$$
\begin{aligned}
& (\mathfrak{A} \mathfrak{B}) X=\mathfrak{A}(\mathfrak{B} X) \quad \forall \mathfrak{A}, \mathfrak{B} \in(\mathfrak{I}), X \in(L) \\
& \left.\mathfrak{A} X \leqq X \quad \forall \mathfrak{A} \in(\mathfrak{I}), X \in(L) \quad{ }^{2}\right) \\
& \left(\bigcup_{i \in I}^{\cup} \mathfrak{A}_{i}\right)\left(\bigcup_{j \in J} X_{j}\right)=\underset{\substack{i \in I \\
j \in J}}{\cup} \mathfrak{A}_{i} X_{j} \quad \forall \mathfrak{A}_{i} \in(\mathfrak{T}), X_{i} \in(L), I, J \text { Indexmengen. }
\end{aligned}
$$

Definition 1. 2. Ist $\mathfrak{A} \in(\mathfrak{I})$ bzw. $Y \in(L)$, so nennt man das größte Element der Menge $\{Z \in(L) ; \mathfrak{A} Z \leqq X\}$ den Rechtsquotienten von $X$ bez. $\mathfrak{A}$ bzw. das größte Element der Menge $\{\mathfrak{B} \in(\mathfrak{T}) ; \bar{B} Y \leqq X\}$ den Linksquotienten von $X$ bez. Y. - Beide Quotienten existieren wegen $(\mathrm{C})$, da $(\bar{L})$ und $(\mathfrak{T})$ vollständig sind.

Schreibweisen:

$\mathfrak{L}(X, Y):=($ Linksquotient von $X$ bez. $Y)=\sup \{\mathfrak{B} \in(\mathfrak{T}) ; \mathfrak{B} Y \leqq X\}$

$R(X, \mathfrak{A}):=($ Rechtsquotient von $X$ bez. $\mathfrak{A})=\sup \{Z \in(L) ; \mathfrak{A} Z \leqq X\}$.

Nach [3] kann man dann zeigen, daß in jeder $n a-(\mathfrak{T})$-Algebra $(L)$ der folgende Satz gilt:

Satz 1. 1. In einer na-(T)-Algebra (L) gelten für beliebige $X, Y, Z \in(L)$ und beliebige $\mathfrak{A}, \mathfrak{B} \in(\mathfrak{I})$ :

$$
\begin{array}{lll}
\text { Q1 } & \mathfrak{U} \leqq \mathfrak{L}(X, X) & \left.X \leqq R(X, \mathfrak{U}){ }^{3}\right) \\
\mathbf{Q 2} & \mathfrak{A} \leqq \mathfrak{L}(X, R(X, \mathfrak{A})) & X \leqq R(Y, \mathfrak{L}(Y, X)) \\
\mathbf{Q 3} & \mathfrak{L}(X, Z) \cap \mathfrak{L}(Y, Z)=\mathfrak{L}(X \cap Y, Z) \\
& R(X, \mathfrak{A}) \cap R(Y, \mathfrak{A})=R(X \cap Y, \mathfrak{A}) \\
\text { Q4 } & \mathfrak{L}(X, Y) \cap \mathfrak{L}(X, Z)=\mathfrak{L}(X, Y \cup Z) \\
& R(X, \mathfrak{A}) \cap R(X, \mathfrak{B})=R(X, \mathfrak{A} \cup \mathfrak{B}) .
\end{array}
$$

Diese Aussagen lassen sich direkt aus der Definition herleiten. Auch die beiden folgenden Aussagen Q5 und Q6 kann man aus dieser beweisen, sie folgen aber auch aus Q3 und Q4:

$$
\text { Q5 } \quad \begin{aligned}
& X \leqq Y \Rightarrow \mathfrak{L}(X, Z) \leqq \mathfrak{L}(Y, Z) \\
& X \leqq Y \Rightarrow R(X, \mathfrak{A}) \leqq R(Y, \mathfrak{U}) \\
& \text { Q6 } \quad Y \leqq Z \Rightarrow \mathfrak{L}(X, Y) \geqq \mathfrak{L}(X, Z) \\
& \\
& \mathfrak{A} \leqq \mathfrak{B} \Rightarrow R(X, \mathfrak{A}) \geqq R(X, \mathfrak{B}),
\end{aligned}
$$

denn aus $Y \leqq Z$ z. B. folgt $Z=Y \cup Z$, also ist

$$
\mathfrak{L}(X, Z)=\mathfrak{L}(X, Y \cup Z)=\mathfrak{L}(X, Y) \frown \mathfrak{L}(X, Z) \leqq \mathfrak{L}(X, Y) .
$$

Im folgenden sollen die im Satz 1.1 bewiesenen Aussagen $\mathbf{Q 1}$ bis $\mathbf{Q 4}$ als definierende Eigenschaften eines neuen Begriffes verwendet werden.

Definition 1. 3. Es seien $(L)$ ein Verband und $(\mathfrak{T})$ ein Verbandsgruppoid. Wir nennen dann $(L)$ ein $(\mathfrak{T})$-System, wenn zu je zwei Elementen $X, Y \in(L)$ und zu jedem Element $\mathfrak{A} \in(\mathfrak{I})$ ein eindeutig bestimmtes Linksresiduum $l(X, Y) \in(\mathfrak{I})$ von $X$ bez. $Y$ und ein eindeutig bestimmtes Rechtsresiduum $r(X, \mathfrak{A}) \in(L)$ von $X$ bez. $\mathfrak{A}$ existieren,

2) Schreibweise: „A<B“ für „, $A \leqq B$ und $A \neq B$ ".

3) Auch aus dieser Bedingung folgt, $\mathrm{da} B(\mathfrak{T})$ ein größtes Element besitzt. Ist ferner $U$ das größte Element von $(L)$, so ist $U<R(U, \mathfrak{A}) \forall \mathfrak{A} \epsilon(\mathfrak{I})$, also $R(U, \mathfrak{U})=U$. 
so daß mit $R(X, \mathfrak{A})=r(X, \mathfrak{A})$ und $\mathfrak{L}(X, Y)=l(X, Y)$ die Aussagen Q1, Q2, Q3 und Q4 gelten.

Bemerkung 1. 1. Ein so definiertes (IT)-System unterscheidet sich von einem in [1] definierten "System mit Residuen" dadurch, daß in einem System mit Residuen der Verband $(\mathfrak{T})$ nicht notwendig ein Verbandsgruppoid ist.

Definition 1. 4. Ein (I)-System $(L)$ heißt $(L)$-noethersch bzw. $(L)$-semimodular, wenn der Verband $(L)$ die aufsteigende Kettenbedingung erfüllt bzw. semimodular ${ }^{4}$ ) ist.

Ein (I)-System heißt (I)-quasi-ganz, wenn das Verbandsgruppoid (T) quasi-ganz ist.

Definition 1. 5. Ein Element $X$ eines (T)-Systems $(L)$ heißt $\frown$-irreduzibel, falls aus $X=Y \cap Z$ mit $Y, Z \in(L)$ und $X \neq Y$ stets $X=Z$ folgt $^{5}$ ).

Von besonderer Wichtigkeit erweist sich hier der folgende Satz:

Satz 1. 2. In einem $(L)$-noetherschen $(\mathfrak{I})$-System $(L)$ ist jedes Element aus $(L)$ als Durchschnitt endlich vieler $\cap$-irreduzibler Elemente darstellbar.

Der Beweis dieses Satzes verläuft analog zu dem 3. Teilerkettensatz von van der Waerden in [10].

\section{§ 2. Tertiäre und primäre Elemente}

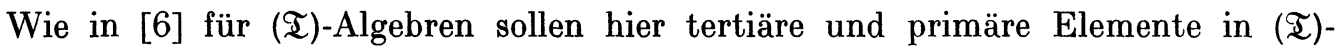
Systemen definiert und einige Eigenschaften gezeigt werden.

Definition 2. 1. Ein Element $T$ eines (I)-Systems $(L)$, zu dem es ein $Y \in(L)$ mit $T<Y$ gibt, heißt tertiär, falls für jedes $X \in(L)$ und jedes $\mathfrak{A} \in(\mathfrak{T})$ mit $r(T, \mathfrak{A}) \frown X=T$ und $T<r(T, \mathfrak{U})$ folgt, daß $X=T$ ist.

Bemerkung 2. 1. Falls ein (T)-System $(L)$ ein größtes Element $U$ besitzt, so soll auch dieses tertiär sein, denn aus der Definition 2. 1 ersieht man, daß jedes $\frown$-irreduzible Element $X \neq U$ aus $(L)$ tertiär ist.

Bemerkung 2. 2. Nach [5] gilt in einem semimodularen Verband $(L)$ :

Aus $R, S \in(L)$ mit $R<S$ folgt die Äquivalenz folgender Aussagen:

(I) Für jedes $X \in(L)$ mit $S \cap X=R$ gilt $X=R$.

(II) Für jedes $Y \in(L)$ mit $S \cap Y \leqq R$ gilt $Y \leqq R$.

Daher ist in einem semimodularen (T)-System $(L)$ die Definition 2.1 äquivalent mit der folgenden:

Definition 2. 2. Ein Element $T$ eines semimodularen (T)-Systems $(L)$, zu dem es ein $Y \in(L)$ mit $T<Y$ gibt, heißt tertiär, falls für jedes $X \in(L)$ und jedes $\mathfrak{A} \in(\mathfrak{T})$ mit $r(T, \mathfrak{A}) \frown X \leqq T$ und $T<r(T, \mathfrak{A})$ folgt, daß $X \leqq T$ ist.

$\mathrm{Da}$ in einem $(\mathfrak{I})$-System eine Potenz $\mathfrak{A}^{n}$ von $\mathfrak{U} \in(\mathfrak{I})$ im allgemeinen erst durch die Angabe eines „Beklammerungstyps“ eindeutig bestimmt ist, soll hier die von E. A.

4) Ein Verband $(L)$ heißt semimodular, wenn es zu je drei Elementen $X, Y, Z \in(L)$ mit

$$
Y \cap Z<X<Z<Y \cup Z
$$

stets ein $T \in(L)$ gibt mit $Y \cap Z<T \leqq Y$ und $X=(X \smile T) \cap Z$. - Diese Definition stimmt mit der in [9] gewählten Definition eines ,halbmodularen“ Verbandes überein. Dort wird auch bewiesen, daß ein Verband endlicher Länge genau dann semimodular ist, wenn er ein Birkhoffscher Verband ist.

$\left.{ }^{5}\right)$ Besitzt $(L)$ ein größtes Element $U$, so ist insbesondere $U$ n-irreduzibel. 
Behrens in [2] eingeführte Schreibweise gewählt werden: , $\Pi_{n}^{(\nu)}(\mathfrak{A})^{“ 6}$ bedeutet dann $\mathfrak{A}^{n}$ bei einer Beklammerung vom Typ $v$.

Definition 2. 3. Ein Element $P$ eines (T)-Systems $(L)$ heißt primär, wenn es für jedes $\mathfrak{U} \in(\mathfrak{T})$, für das ein $X \in(L)$ mit $X \leqq r(P, \mathfrak{A})$ und $X \leqq P$ existiert, ein $n \in N$ und einen Typ $v$ gibt, so daß gilt:

$$
\left.r\left(Y, \Pi_{n}^{(v)}(\mathfrak{A})\right) \leqq r\left(P, \Pi_{n}^{(v)}(\mathfrak{A})\right) \quad \forall Y \in(L) \quad{ }^{6}\right) .
$$

$\mathrm{Zu}$ der Definition 2. 3 wollen wir hier noch eine äquivalente Aussage formulieren.

Satz 2. 1. Sei $(L)$ ein (I)-System und $P$ ein beliebiges Element aus $(L)$. Dann sind die beiden folgenden Aussagen äquivalent:

A $P$ ist primär.

B $Z u$ jedem $\mathfrak{A} \in(\mathfrak{T})$ mit $P<r(P, \mathfrak{A})$ gibt es ein $n \in N$ und einen Typ $v$ mit

$$
r\left(Y, \Pi_{n}^{(\nu)}(\mathfrak{U})\right) \leqq r\left(P, \Pi_{n}^{(v)}(\mathfrak{A})\right) \quad \forall Y \in(L) .
$$

Der Beweis dieses Satzes verläuft hier analog zu dem in [11], Satz 2.1 angegebenen Beweis.

Definition 2. 4. Wir wollen sagen, daß ein Element $X$ eines (T)-Systems $(L)$ eine Primär- bzw. Tertiärdarstellung besitzt, falls sich $X$ als Durchschnitt endlich vieler primärer bzw. tertiärer Elemente aus $(L)$ darstellen läßt.

Im folgenden wollen wir noch direkte Beziehungen zwischen tertiären und primären Elementen angeben.

Satz 2. 2. Ist in einem (T)-System $(L)$ jedes tertiäre Element aus $(L)$ primär, so ist auch jedes $\frown$-irreduzible Element aus $(L)$ primär.

Beweis. Nach Bemerkung 2.1 ist jedes $\frown$-irreduzible Element aus $(L)$ tertiär, nach Voraussetzung also primär.

Satz 2. 3. Ist in einem $(L)$-noetherschen $(\mathfrak{I})$-System $(L)$ jedes $\frown$-irreduzible Element aus $(L)$ primär, so besitzt jedes Element aus $(L)$ eine Primärdarstellung.

Beweis. Nach Satz 1. 2 läßt sich in einem $(L)$-noetherschen (T)-System jedes Element aus $(L)$ als Durchschnitt endlich vieler $\cap$-irreduzibler Elemente aus $(L)$ darstellen. Damit folgt aber aus der Voraussetzung dieses Satzes sofort die Behauptung.

\section{§ 3. Die Bedingung von Artin und Rees}

In noetherschen Ringen, aber auch in noetherschen $R$-Moduln kann man das „Lemma von E. Artin und D. Rees" beweisen (vgl. etwa [8]) ${ }^{7}$ ).

Dieses Lemma erhielt bei Kurata in [4] eine neue Bedeutung, indem sich die Gültigkeit dieses Lemmas als äquivalent zu der Aussage erwies, daß jedes $\frown$-irreduzible zweiseitige Ideal rechtsprimär (und nicht nur rechtstertiär) ist. Diese Bedeutung erhält das Lemma von Artin und Rees in einer verallgemeinerten Form auch in der Theorie der

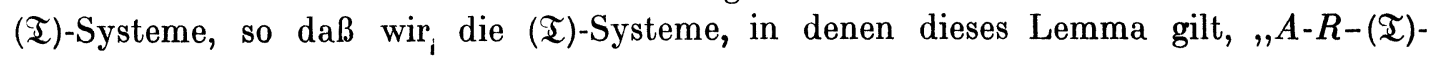
Systeme" nennen wollen.

6) Besitzt $(L)$ ein größtes Element $U$, so ist $U$ nach dieser Definition primär.

$\left.{ }^{7}\right)$ Das Lemma von Artin und Rees besagt, daß in einem noetherschen Ring $R$ zu je zwei Idealen $\mathfrak{a}, \mathfrak{b}$ aus $R$ und zu jeder natürlichen Zahl $n$ eine natürliche Zahl $m$ existiert, so daß $\mathfrak{a}^{m} \cap \mathfrak{b} \leqq \mathfrak{a}^{n} \cdot \mathfrak{b}$ gilt. 
Definition 3. 1. Existieren in einem (T)-System $(L)$ für jedes $\mathfrak{A} \in(\mathfrak{I})$, jedes $Z \in(L)$, jedes $n \in N$ und jeden Typ $v$ einer Beklammerung ein Element $V \in(L)$, ein $m \in N$ und ein Beklammerungstyp $\mu$, so daß gilt:

$$
V \frown r\left(Z, \Pi_{n}^{(v)}(\mathfrak{A})\right) \leqq Z \text { und } r\left(V, \Pi_{m}^{(\mu)}(\mathfrak{A})\right) \geqq r\left(Y, \Pi_{m}^{(\mu)}(\mathfrak{A})\right) \quad \forall Y \in(L),
$$

so nennt man $(L)$ ein $A-R-(\mathfrak{T})$-System.

Es läßt sich nun zeigen, daß sogar eine spezielle Form eines $A-R-(\mathfrak{T})$-Systems in bestimmten Fällen dieselbe Bedeutung erlangt wie ein $A-R-(\mathfrak{T})$-System:

Definition 3. 2. Existieren in einem (T)-System $(L)$ für jedes $\mathfrak{A} \in(\mathfrak{T})$ und jedes $Z \in(L)$ ein Element $V \in(L)$, ein $m \in N$ und ein Beklammerungstyp $\mu$, so daß gilt:

$$
V \cap r(Z, \mathfrak{A}) \leqq Z \text { und } r\left(V, \Pi_{m}^{(\mu)}(\mathfrak{A})\right) \geqq r\left(Y, \Pi_{n}^{(\mu)}(\mathfrak{A})\right) \quad \forall Y \in(L),
$$

so nennt man $(L)$ ein Quasi-A-R-(T)-System.

Bemerkung 3. 1. Jedes $A-R-(\mathfrak{T})$-System ist ein Quasi- $A-R-(\mathfrak{T})$-System.

In dem folgenden Satz 3.1 soll ein Zusammenhang zwischen einem $A-R-(\mathfrak{T})$ System und einem $(\mathfrak{T})$-System $(L)$, in dem jedes Element aus $(L)$ eine Primärdarstellung besitzt, aufgezeigt werden. Weitgehend wird dabei die Schlußweise von Kurata [4] benutzt.

Lemma 3. 1. Sei $(L)$ ein (T)-System mit einem größten Element $U$. Ist dann $P$ ein primäres Element aus $(L)$ und ist $\mathfrak{A}$ ein Element aus $(\mathfrak{I})$, zu dem es ein $X \in(L)$ mit $X \leqq r(P, \mathfrak{A})$ und $X \leqq P$ gibt, so existieren ein $n \in N$ und ein Typ $v$ mit

$$
r\left(P, \Pi_{n}^{(v)}(\mathfrak{A})\right)=U .
$$

Beweis. Nach Definition 2. 3 gilt unter den gegebenen Voraussetzungen

$$
r\left(Y, \Pi_{n}^{(v)}(\mathfrak{A})\right) \leqq r\left(P, \Pi_{n}^{(v)}(\mathfrak{A})\right) \quad \forall Y \in(L) .
$$

Folglich ist nach $\mathbf{Q 1}$ und Definition von $U$ auch

$$
U \leqq r\left(U, \Pi_{n}^{(v)}(\mathfrak{A})\right) \leqq r\left(P, \Pi_{n}^{(v)}(\mathfrak{U})\right) \leqq U,
$$

woraus die Behauptung sofort folgt.

Satz 3. 1. Sei $(L)$ ein (I)-quasi-ganzes (I)-System mit einem größten Element $U$. Besitzt dann jedes Element aus $(L)$ eine Primärdarstellung, so ist $(L)$ ein $A-R-(\mathfrak{I})$ System.

Bewcis. Seien $\mathfrak{A} \in(\mathfrak{T}), Z \in(L), n \in N$ und ein Typ $\nu$ beliebig gewählt. Wir setzen dann

$$
X=r\left(Z, \Pi_{n}^{(v)}(\mathfrak{A})\right)
$$

Nach Voraussetzung besitzt $Z$ eine Primärdarstellung:

$$
Z=P_{1} \cap P_{2} \cap \cdots \cap P_{r} .
$$

Zu zeigen ist, daß ein $V \in(L)$, ein $m \in N$ und ein Typ $\mu$ existieren mit $V \frown X \leqq Z$ und

$$
r\left(Y, \Pi_{m}^{(\mu)}(\mathfrak{A})\right) \leqq r\left(V, \Pi_{m}^{(\mu)}(\mathfrak{U})\right) \quad \forall Y \in(L) .
$$

Ist nun $X \leqq P_{1}, P_{2}, \ldots, P_{r}$, so gilt $X \leqq Z$. Daher ist für jedes beliebig gewählte Element $V \in(L)$ bereits $V \cap X \leqq Z$.

Nach Voraussetzung besitzt $(L)$ ein größtes Element $U$. Setzen wir nun $U=V$, so ist auch $r(Y, \mathfrak{A}) \leqq r(V, \mathfrak{A})$ für jedes $Y \in(L)$ nach $\mathbf{Q 5}$ erfüllt, d. h. $(L)$ ist in diesem Fall $A-R-(\mathfrak{I})-$ System. 
Gibt es jedoch ein $i$ mit $X \$ P_{i}$, so kann man wegen der Unabhängigkeit der Darstellung (A) von der Reihenfolge voraussetzen, daß die $P_{i}$ so numeriert sind, daß es ein $s$ mit $1 \leqq s \leqq r$ gibt, so daß $X \npreceq P_{1}, P_{2}, \ldots, P_{s}$ und $X \leqq P_{s+1}, \ldots, P_{r}$ gilt, falls $s<r$ ist. Dann gilt: $\left(P_{1} \cap P_{2} \cap \cdots \cap P_{s}\right) \cap X \leqq Z$. Nach (A) gilt nun $Z \leqq P_{i}$ für jedes $i$, nach $\mathbf{Q 5}$ also

$$
X=r\left(Z, \Pi_{n}^{(v)}(\mathfrak{Q})\right) \leqq r\left(P_{i}, \Pi_{n}^{(v)}(\mathfrak{X})\right) .
$$

Da die Elemente $P_{i}$ primär sind, folgt nach Lemma 3. 1 aus $X \leqq r\left(P_{i}, \Pi_{n}^{(\nu)}(\mathfrak{Q})\right)$ und $X \not P_{i}$ für $i \in\{1, \ldots, s\}$, daß für jedes $i \in\{1, \ldots, s\}$ ein $m_{i} \in N$ und ein Typ $\mu_{i}$ existieren mit

$$
U=r\left(P_{i}, \Pi_{m_{i}}^{\left(\mu_{i}\right)}\left(\Pi_{n}^{(v)}(\mathfrak{A})\right)\right)
$$

Also gibt es Typen $\lambda_{i}$, für die gilt:

$$
U=r\left(P_{i}, \Pi_{n m_{i}}^{\left(\lambda_{i}\right)}(\mathfrak{A})\right)
$$$$
i \in\{1, \ldots, s\} \text {. }
$$

Da $(L)$ nach Voraussetzung $(\mathfrak{T})$-quasi-ganz ist, gilt auch

$$
\Pi_{n m_{1}}^{\left(\lambda_{1}\right)}(\mathfrak{U}) \cdot\left(\Pi_{n m_{2}}^{\left(\lambda_{2}\right)}(\mathfrak{U}) \cdot\left(\cdots \Pi_{n m_{g}}^{\left(\lambda_{g}\right)}(\mathfrak{U})\right)\right) \leqq \Pi_{n m_{i}}^{\left(\lambda_{i}\right)}(\mathfrak{A})
$$

für jedes $i$ mit $1 \leqq i \leqq s$. Folglich gibt es zu $m:=\sum_{i=1}^{8} n m_{i}$ einen Typ $\mu$ mit

$$
\Pi_{m}^{(\mu)}(\mathfrak{U}) \leqq \Pi_{n m_{i}}^{\left(\lambda_{i}\right)}\left(\mathfrak{A}_{i}\right) \quad \forall i \in\{1, \ldots, s\} .
$$

Nach Q6 und (B) ist daher auch

$$
U=r\left(P_{i}, \Pi_{n m_{i}}^{\left(\lambda_{i}\right)}(\mathfrak{U})\right) \leqq r\left(P_{i}, \Pi_{m}^{(\mu)}(\mathfrak{U})\right)
$$

also, da $U$ größtes Element aus $(L)$ ist,

$$
r\left(P_{i}, \Pi_{m}^{(\mu)}(\mathfrak{A})\right)=U \text { für } i \in\{1, \ldots, s\} .
$$

Nach Q3 ist dann auch

$$
U=r\left(P_{1} \cap P_{2} \cap \cdots \cap P_{s}, \Pi_{m}^{(\mu)}(\mathfrak{A})\right) .
$$

Folglich hat man mit $V=P_{1} \cap \cdots \cap P_{s}$ sowohl die Existenz eines $m \in N$ wie eines Typs $\mu$ mit

also auch

$$
r\left(Y, \Pi_{m}^{(\mu)}(\mathfrak{Q})\right) \leqq U=r\left(V, \Pi_{m}^{(\mu)}(\mathfrak{A})\right) \quad \forall Y \in(L),
$$

gezeigt.

$$
V \cap X \leqq P_{1} \cap \cdots \cap P_{s} \cap \cdots \cap P_{r}=Z
$$

Mit Hilfe von Satz 3.1 ist jetzt bewiesen, daß ein (I) -quasi-ganzes, $(L)$-noethersches (T)-System $(L)$ mit einem größten Element, in dem nach Satz 1.2 jedes Elernent aus $(L)$ als Durchschnitt endlich vieler $\frown$-irreduzibler Elemente darstellbar ist, ein $A-R-(\mathfrak{T})$-System ist, falls jedes $\frown$-irreduzible Element primär ist. In dem folgenden Satz soll nun gezeigt werden, daß in einem $(L)$-semimodularen (T)-System diese Aussage auch umkehrbar ist, d. h. in einem $(L)$-semimodularen $A$ - $R$-(T)-System jedes $\cap$-irreduzible Element primär ist. Nach der Bemerkung 3.1 sowie nach Satz 2.2 genügt es, dafür den folgenden Satz zu beweisen:

Satz 3. 2. (L) sei ein (L)-semimodulares Quasi-A-R-(T)-System. Dann ist jedes tertiäre Element aus $(L)$ primär.

Beweis. Sei $T$ ein tertiäres Element aus $(L)$. Es soll gezeigt werden, daß $T$ die Aussage $\mathbf{B}$ in Satz 2.1 erfüllt. Gibt es kein $\mathfrak{U} \in(\mathfrak{T})$ mit $T<r(T, \mathfrak{U})$, so ist $T$ primär. 
Sei nun $\mathfrak{A} \in(\mathfrak{I})$ mit $T<r(T, \mathfrak{U})$. -

Da $(L)$ ein Quasi- $A-R-(\mathfrak{T})$-System ist, gibt es ein Element $V \in(L)$, ein $m \in N$ und einen Typ $\mu$, so daß gilt:

und

$$
r\left(Y, \Pi_{m}^{(\mu)}(\mathfrak{U})\right) \leqq r\left(V, \Pi_{m}^{(\mu)}(\mathfrak{U})\right) \quad \forall Y \in(L)
$$

$$
V \frown r(T, \mathfrak{A}) \leqq T
$$

Da $T$ tertiär und $(L)$ sogar $(L)$-semimodular ist, folgt nach Definition aus

$$
V \frown r(T, \mathfrak{A}) \leqq T \text { und } T<r(T, \mathfrak{A})
$$

bereits $V \leqq T$, also $r\left(T, \Pi_{m}^{(\mu)}(\mathfrak{A})\right) \geqq r\left(V, \Pi_{m}^{(\mu)}(\mathfrak{A})\right) \geqq r\left(Y, \Pi_{m}^{(\mu)}(\mathfrak{H})\right)$ für jedes $Y \in(L)$ nach Q5, d. h. $T$ ist primär.

\section{§ 4. Die verallgemeinerte Kurata-Äquivalenz}

Als Ergebnis können wir jetzt den folgenden Satz als Folgerung von Satz 3. 1, Bemerkung 3. 1, Satz 3. 2, Satz 2. 2 und Satz 2. 3 fomulieren:

Satz 4. 1. Ist $(L)$ ein $(L)$-noethersches, $(L)$-semimodulares und (I)-quasi-ganzes $(\mathfrak{T})-$ System, in dem der Verband $(L)$ ein größtes Element besitzt, so sind die folgenden Aussagen äquivalent:
A Jedes Element aus (L) besitzt eine Primärdarstellung.
B (L) ist ein $A-R-(\mathfrak{T})-S y s t e m$.
C $(L)$ ist ein Quasi-A-R-(T)-System.
D Jedes tertiäre Element aus $(L)$ ist primär.
E Jedes $\frown$-irreduzible Element aus $(L)$ ist primür.

\section{Literatur}

[1] V. A. Andrunakievic, Ju. M. Rjabuhin, Additive theory of ideals in systems with residuals (rassisch), lzv. Akad. Nauk SSSR Ser. Mat. 31 (1967), 1057-1090; engl. Übers. in Math. USSR-Izvestija 1 (1967), 1011-1040.

[2] E.-A. Behrens, Zur additiven Idealtheorie in nicht-assoziativen Ringen, Math. Zeit. 64 (1956), 169-182.

[3] M. L. Dubreil-Jacotin, L. Lesieur, R. Croisot, Leçons sur la Théorie des Treillis de Structures Algébriques Ordonnées et des Treillis Géométriques, Paris 1953.

[4] Y. Kurata, On an additive ideal theory in a non-associative ring, Math. Zeit. 88 (1965), 129-135.

[5] L. Lesieur, $R$, Croisot, Théorie noethérienne des anneaux, des demi-groupes et des modules dans le cas non commutatif. I: Colloque d'Algèbre Supérieure, CBRM, Bruxelles 1956, 79-121.

[6] L. Lesieur, R. Croisot, Algèbre noethérienne non commutative. Mémorial des Sciences mathématiques, Paris 1963.

[7] L. Lesieur, R. Croisot, Extension au cas non commutatif d'un théorème de Krull et d'un lemme d'Artin-Rees, J. reine angew. Math. 204 (1960), 216-220.

[8] D. G. Northcott, Lessons on Rings, Modules and Multiplicities, Cambridge 1968.

[9] G. Szasz, Einführung in die Verbandstheorie, Budapest 1962.

[10] B. L. van der Waerden, Algebra II, Berlin, Göttingen, Heidelberg, 4. Aufl. 1959.

[11] D. Windelberg, Zur additiven Idealtheorie in nicht-assoziativen (T)-Algebren, J. reine angew. Math. 239-240 (1970), 180-184.

TU Hannover, Lehrstuhl für Geometrie, 3 Hannover, Welfengarten 1 